\title{
Research Article \\ On the Stability of Cubic Mappings and Quadratic Mappings in Random Normed Spaces
}

\author{
E. Baktash, ${ }^{1}$ Y. J. Cho, ${ }^{2}$ M. Jalili, ${ }^{3}$ R. Saadati, ${ }^{4,5}$ and S. M. Vaezpour ${ }^{4}$ \\ ${ }^{1}$ Youngs Researchers Club and Department of Basic Sciences, Islamic Azad University, \\ Ayatollah Amoli Branch, P.O. Box 678, Amol, Iran \\ ${ }^{2}$ Department of Mathematics Education and the RINS, Gyeongsang National University, \\ Chinju 660-701, South Korea \\ ${ }^{3}$ Department of Mechanical Engineering, Islamic Azad University, Ayatollah Amoli Branch, \\ P.O. Box 678, Amol, Iran \\ ${ }^{4}$ Department of Mathematics and Computer Science, Amirkabir University of Technology, \\ 424 Hafez Avenue, Tehran 15914, Iran \\ ${ }^{5}$ Faculty of Sciences, University of Shomal, P.O. Box 731, Amol, Iran
}

Correspondence should be addressed to Y. J. Cho, yjcho@gsnu.ac.kr

Received 27 August 2008; Revised 23 October 2008; Accepted 24 November 2008

Recommended by Wing-Sum Cheung

Recently, the stability of the cubic functional equation $f(2 x+y)+f(2 x-y)=2 f(x+y)+2 f(x-$ $y)+12 f(x)$ in fuzzy normed spaces was proved in earlier work; and the stability of the additive functional equations $f(x+y)=f(x)+f(y), 2 f((x+y) / 2)=f(x)+f(y)$ in random normed spaces was proved as well. In this paper, we prove the stability of the cubic functional equation $f(2 x+y)+f(2 x-y)=2 f(x+y)+2 f(x-y)+12 f(x)$ in random normed spaces by an alternative proof which provides a better estimation. Finally, we prove the stability of the quartic functional equation $f(2 x+y)+f(2 x-y)=4 f(x+y)+4 f(x-y)+24 f(x)-6 f(y)$ in random normed spaces.

Copyright (C) 2008 E. Baktash et al. This is an open access article distributed under the Creative Commons Attribution License, which permits unrestricted use, distribution, and reproduction in any medium, provided the original work is properly cited.

\section{Introduction and preliminaries}

The study of stability problems for functional equations is related to a question of Ulam [1] concerning the stability of group homomorphisms and affirmatively answered for Banach spaces by Hyers [2]. Subsequently, the result of Hyers was generalized by Aoki [3] for additive mappings and by Th. M. Rassias [4] for linear mappings by considering an unbounded Cauchy difference. The paper of Th. M. Rassias has provided a lot of influence in the development of what we now call Hyers-Ulam-Rassias stability of functional equations. We refer the interested readers for more information on such problems to the papers [5-9]. The functional equation

$$
f(2 x+y)+f(2 x-y)=2 f(x+y)+2 f(x-y)+12 f(x)
$$


is said to be the cubic functional equation since the function $f(x)=c x^{3}$ is its solution. Every solution of the cubic functional equation is said to be a cubic mapping. The stability problem for the cubic functional equation was proved by Jun and Kim [10] for mappings $f: X \rightarrow Y$, where $X$ is a real normed space and $Y$ is a Banach space. Later, a number of mathematicians have worked on the stability of some types of the cubic equation [11]. The functional equation

$$
f(2 x+y)+f(2 x-y)=4 f(x+y)+4 f(x-y)+24 f(x)-6 f(y)
$$

is said to be the quadratic functional equation since the function $f(x)=c x^{4}$ is its solution. Every solution of the quadratic functional equation is said to be a quadratic mapping. The stability problem for the quadratic functional equation first was proved by J. M. Rassias [12] for mappings $f: X \rightarrow Y$, where $X$ is a real normed space and $Y$ is a Banach space. In addition, Mirmostafaee et al. [13-15], Alsina [16], Miheţ and Radu [17] investigated the stability in the settings of fuzzy, probabilistic, and random normed spaces.

In the sequel, we will adopt the usual terminology, notations, and conventions of the theory of random normed spaces as in [17-21]. Throughout this paper, the space of all probability distribution functions is denoted by

$$
\begin{aligned}
& \Delta^{+}=\{F: \mathbb{R} \cup\{-\infty,+\infty\} \longrightarrow[0,1]: F \text { is left-continuous } \\
&\text { and nondecreasing on } \mathbb{R} \text { and } F(0)=0, F(+\infty)=1\},
\end{aligned}
$$

and the subset $D^{+} \subseteq \Delta^{+}$is the set $D^{+}=\left\{F \in \Delta^{+}: l^{-} F(+\infty)=1\right\}$, where $l^{-} f(x)$ denotes the left limit of the function $f$ at the point $x$. The space $\Delta^{+}$is partially ordered by the usual pointwise ordering of functions, that is, $F \leq G$ if and only if $F(t) \leq G(t)$ for all $t \in \mathbb{R}$. The maximal element for $\Delta^{+}$in this order is the distribution function given by

$$
\varepsilon_{0}(t)= \begin{cases}0, & \text { if } t \leq 0 \\ 1, & \text { if } t>0\end{cases}
$$

Definition 1.1 (see [17]). A function $T:[0,1] \times[0,1] \rightarrow[0,1]$ is a continuous triangular norm (briefly, a $t$-norm) if $T$ satisfies the following conditions:

(a) $T$ is commutative and associative;

(b) $T$ is continuous;

(c) $T(a, 1)=a$ for all $a \in[0,1]$;

(d) $T(a, b) \leq T(c, d)$ whenever $a \leq c$ and $b \leq d$ for all $a, b, c, d \in[0,1]$.

Three typical examples of continuous $t$-norms are $T(a, b)=a b, T(a, b)=\max (a+b-$ $1,0)$, and $T(a, b)=\min (a, b)$.

Definition 1.2. A random normed space (briefly, $\mathrm{RN}$-space) is a triple $(X, \mu, T)$, where $X$ is a vector space, $T$ is a continuous $t$-norm, and $\mu$ is a mapping from $X$ into $D^{+}$such that 
the following conditions hold:

(PN1) $\mu_{x}(t)=\varepsilon_{0}(t)$ for all $t>0$ if and only if $x=0$;

(PN2) $\mu_{\alpha x}(t)=\mu_{x}(t /|\alpha|)$ for all $x$ in $X, \alpha \neq 0$ and all $t \geq 0$;

(PN3) $\mu_{x+y}(t+s) \geq T\left(\mu_{x}(t), \mu_{y}(s)\right)$ for all $x, y \in X$ and all $t, s \geq 0$.

Definition 1.3. Let $(X, \mu, T)$ be an $\mathrm{RN}$-space.

(1) A sequence $\left\{x_{n}\right\}$ in $X$ is said to be convergent to $x$ in $X$ if, for every $t>0$ and $\varepsilon>0$, there exists a positive integer $N$ such that $\mu_{x_{n}-x}(t)>1-\varepsilon$ whenever $n \geq N$.

(2) A sequence $\left\{x_{n}\right\}$ in $X$ is called a Cauchy sequence if, for every $t>0$ and $\varepsilon>0$, there exists a positive integer $N$ such that $\mu_{x_{n}-x_{m}}(t)>1-\varepsilon$ whenever $n \geq m \geq N$.

(3) An RN-space $(X, \mu, T)$ is said to be complete if and only if every Cauchy sequence in $X$ is convergent to a point in $X$.

Theorem 1.4 (see [20]). If $(X, \mu, T)$ is an RN-space and $\left\{x_{n}\right\}$ is a sequence such that $x_{n} \rightarrow x$, then $\lim _{n \rightarrow \infty} \mu_{x_{n}}(t)=\mu_{x}(t)$.

Lemma 1.5. Let $(X, \mu, \min )$ be an $R N$-space and define $E_{\lambda, \mu}: X \rightarrow \mathbb{R}^{+} \cup\{0\}$ by

$$
\left.E_{\lambda, \mu}(x)=\inf \left\{t>0: \mu_{x}(t)>1-\lambda\right\}, \quad \forall \lambda \in\right] 0,1[, x \in X
$$

Then, one has

$$
E_{\lambda, \mu}\left(x_{1}-x_{n}\right) \leq E_{\lambda, \mu}\left(x_{1}-x_{2}\right)+\cdots+E_{\lambda, \mu}\left(x_{n-1}-x_{n}\right),
$$

for all $x_{1}, \ldots, x_{n} \in X$ and the sequence $\left\{x_{n}\right\}$ is convergent to $x$ with respect to random norm $\mu$ if and only if $E_{\lambda, \mu}\left(x_{n}-x\right) \rightarrow 0$ as $n \rightarrow \infty$. Also, the sequence $\left\{x_{n}\right\}$ is a Cauchy sequence with respect to random norm $\mu$ if and only if it is a Cauchy sequence with $E_{\lambda, \mu}$.

Proof. By the triangular inequality, we have

$$
\begin{aligned}
\mu_{x_{1}-x_{n}} & \left(E_{\lambda, \mu}\left(x_{1}-x_{2}\right)+\cdots+E_{\lambda, \mu}\left(x_{n-1}-x_{n}\right)+(n-1) \delta\right) \\
\geq & \min \left(\mu_{x_{1}-x_{2}}\left(E_{\lambda, \mu}\left(x_{1}-x_{2}\right)+\delta\right), \ldots, \mu_{x_{n-1}-x_{n}}\left(E_{\lambda, \mu}\left(x_{n-1}-x_{n}\right)+\delta\right)\right) \\
> & \min (1-\lambda, \ldots, 1-\lambda) \\
\quad & 1-\lambda, \quad \forall \delta>0,
\end{aligned}
$$

which implies that

$$
E_{\lambda, \mu}\left(x_{1}-x_{n}\right) \leq E_{\lambda, \mu}\left(x_{1}-x_{2}\right)+E_{\lambda, \mu}\left(x_{2}-x_{3}\right)+\cdots+E_{\lambda, \mu}\left(x_{n-1}-x_{n}\right)+(n-1) \delta .
$$

Since $\delta>0$ is arbitrary, we have

$$
E_{\lambda, \mu}\left(x_{1}-x_{n}\right) \leq E_{\lambda, \mu}\left(x_{1}-x_{2}\right)+E_{\lambda, \mu}\left(x_{2}-x_{3}\right)+\cdots+E_{\lambda, \mu}\left(x_{n-1}-x_{n}\right) .
$$



proof.

Next, we have $\mu_{x_{n}-x}(\eta)>1-\lambda \Leftrightarrow E_{\lambda, \mu}\left(x_{n}-x\right)<\eta$ for every $\eta>0$. This completes the

In this paper, we establish the stability of the cubic and quadratic functional equations in the setting of random normed spaces.

\section{On the stability of cubic mappings in $\mathrm{RN}$-spaces}

Theorem 2.1. Let $X$ be a linear space, $\left(Z, \mu^{\prime}, \min \right)$ an $R N$-space, and $\varphi: X \times X \rightarrow Z$ a function such that for some $0<\alpha<8$,

$$
\mu_{\varphi(2 x, 0)}^{\prime}(t) \geq \mu_{\alpha \varphi(x, 0)}^{\prime}(t), \quad \forall x \in X, t>0,
$$

$f(0)=0$ and $\lim _{n \rightarrow \infty} \mu_{\varphi\left(2^{n} x, 2^{n} y\right)}^{\prime}\left(8^{n} t\right)=1$ for all $x, y \in X$ and all $t>0$. Let $(Y, \mu, \min )$ be a complete $R N$-space. If $f: X \rightarrow Y$ is a mapping such that

$$
\mu_{f(2 x+y)+f(2 x-y)-2 f(x+y)-2 f(x-y)-12 f(x)}(t) \geq \mu_{\varphi(x, y)}^{\prime}(t), \quad \forall x, y \in X, t>0,
$$

then there exists a unique cubic mapping $C: X \rightarrow Y$ such that

$$
\left.\mu_{f(x)-C(x)}(t) \geq \mu_{\varphi(x, 0)}^{\prime}(2(8-\alpha) t)\right)
$$

Proof. From (2.2), it follows that

$$
\begin{aligned}
E_{\lambda, \mu}( & f(2 x+y)+f(2 x-y)-2 f(x+y)-2 f(x-y)-12 f(x)) \\
& =\inf \left\{t>0: \mu_{f(2 x+y)+f(2 x-y)-2 f(x+y)-2 f(x-y)-12 f(x)}(t)>1-\lambda\right\} \\
& \leq \inf \left\{t>0: \mu_{\varphi(x, y)}^{\prime}(t)>1-\lambda\right\} \\
& =E_{\lambda, \mu^{\prime}}(\varphi(x, y)), \quad \forall x, y \in X, \lambda \in(0,1) .
\end{aligned}
$$

Putting $y=0$ in (2.4), we get

$$
E_{\lambda, \mu}\left(\frac{f(2 x)}{8}-f(x)\right) \leq \frac{1}{16} E_{\lambda, \mu^{\prime}}(\varphi(x, 0)), \quad \forall x \in X
$$

Replacing $x$ by $2^{n} x$ in (2.5) and using (2.1), we obtain

$$
\begin{aligned}
E_{\lambda, \mu}\left(\frac{f\left(2^{n+1} x\right)}{8^{n+1}}-\frac{f\left(2^{n} x\right)}{8^{n}}\right) & \leq \frac{1}{16 \times 8^{n}} E_{\lambda, \mu^{\prime}}\left(\varphi\left(2^{n} x, 0\right)\right) \\
& \leq \frac{\alpha^{n}}{16 \times 8^{n}} E_{\lambda, \mu^{\prime}}(\varphi(x, 0))
\end{aligned}
$$


E. Baktash et al.

It follows from $\left(f\left(2^{n} x\right) / 8^{n}\right)-f(x)=\sum_{k=0}^{n-1}\left(\left(f\left(2^{k+1} x\right) / 8^{k+1}\right)-\left(f\left(2^{k} x\right) / 8^{k}\right)\right)$ and (2.6) that

$$
\begin{aligned}
E_{\lambda, \mu}\left(\frac{f\left(2^{n} x\right)}{8^{n}}-f(x)\right) & =E_{\lambda, \mu}\left(\sum_{k=0}^{n-1}\left(\frac{f\left(2^{k+1} x\right)}{8^{k+1}}-\frac{f\left(2^{k} x\right)}{8^{k}}\right)\right) \\
& \leq \sum_{k=0}^{n-1} E_{\lambda, \mu}\left(\frac{f\left(2^{k+1} x\right)}{8^{k+1}}-\frac{f\left(2^{k} x\right)}{8^{k}}\right) \\
& \leq \sum_{k=0}^{n-1} \frac{1}{16 \times 8^{k}} E_{\lambda, \mu^{\prime}}\left(\varphi\left(2^{k} x, 0\right)\right) \\
& \leq \sum_{k=0}^{n-1} \frac{\alpha^{k}}{16 \times 8^{k}} E_{\lambda, \mu^{\prime}}(\varphi(x, 0)) .
\end{aligned}
$$

Replacing $x$ with $2^{m} x$ in (2.7), we observe that

$$
\begin{aligned}
E_{\lambda, \mu}\left(\frac{f\left(2^{n+m} x\right)}{8^{n+m}}-\frac{f\left(2^{m} x\right)}{8^{m}}\right) & \leq \sum_{k=0}^{n-1} \frac{\alpha^{k}}{16 \times 8^{k+m}} E_{\lambda, \mu^{\prime}}\left(\varphi\left(2^{m} x, 0\right)\right) \\
& \leq \sum_{k=0}^{n-1} \frac{\alpha^{k+m}}{16 \times 8^{k+m}} E_{\lambda, \mu^{\prime}}(\varphi(x, 0)) \\
& \leq \sum_{k=m}^{m+n-1} \frac{\alpha^{k}}{16 \times 8^{k}} E_{\lambda, \mu^{\prime}}(\varphi(x, 0)) \\
& =\frac{E_{\lambda, \mu^{\prime}}(\varphi(x, 0))}{16} \sum_{k=m}^{m+n-1}\left(\frac{\alpha}{8}\right)^{k} .
\end{aligned}
$$

Then $\left\{f\left(2^{n} x\right) / 8^{n}\right\}$ is a Cauchy sequence in $(Y, \mu, \min )$. Since $(Y, \mu, \min )$ is a complete RNspace, this sequence converges to some point $C(x) \in Y$. Fix $x \in X$ and put $m=0$ in (2.8). Then we obtain

$$
E_{\lambda, \mu}\left(\frac{f\left(2^{n} x\right)}{8^{n}}-f(x)\right) \leq \frac{E_{\lambda, \mu^{\prime}}(\varphi(x, 0))}{16} \sum_{k=0}^{n-1}\left(\frac{\alpha}{8}\right)^{k}
$$

and so

$$
\begin{aligned}
E_{\lambda, \mu}(C(x)-f(x)) & \leq E_{\lambda, \mu}\left(C(x)-\frac{f\left(2^{n} x\right)}{8^{n}}\right)+E_{\lambda, \mu}\left(\frac{f\left(2^{n} x\right)}{8^{n}}-f(x)\right) \\
& \leq E_{\lambda, \mu}\left(C(x)-\frac{f\left(2^{n} x\right)}{8^{n}}\right)+\frac{E_{\lambda, \mu^{\prime}}(\varphi(x, 0))}{16} \sum_{k=0}^{n-1}\left(\frac{\alpha}{8}\right)^{k} .
\end{aligned}
$$


Taking the limit as $n \rightarrow \infty$ and using (2.10), we get

$$
E_{\lambda, \mu}(C(x)-f(x)) \leq \frac{E_{\lambda, \mu^{\prime}}(\varphi(x, 0))}{16-2 \alpha}
$$

that is,

$$
\inf \left\{t>0: \mu_{C(x)-f(x)}(t)>1-\lambda\right\} \leq \inf \left\{t>0: \mu_{\varphi(x, 0)}^{\prime}(2 t(8-\alpha))>1-\lambda\right\} .
$$

Then, we have

$$
\mu_{C(x)-f(x)}(t) \geq \mu_{\varphi(x, 0)}^{\prime}(2 t(8-\alpha))
$$

Replacing $x$ and $y$ by $2^{n} x$ and $2^{n} y$ in (2.2), respectively, we get

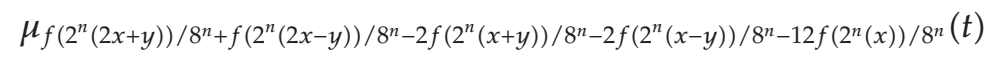

$$
\begin{aligned}
& \geq \mu_{\varphi\left(2^{n} x, 2^{n} y\right)}^{\prime}\left(8^{n} t\right), \quad \forall x, y \in X, t>0 .
\end{aligned}
$$

Since $\lim _{n \rightarrow \infty} \mu_{\varphi\left(2^{n} x, 2^{n} y\right)}^{\prime}\left(8^{n} t\right)=1$, we conclude that $C$ fulfills (1.1).

To prove the uniqueness of the cubic mapping $C$, assume that there exists a cubic mapping $D: X \rightarrow Y$ which satisfies (2.3). Fix $x \in X$. Clearly, $C\left(2^{n} x\right)=8^{n} C(x)$ and $D\left(2^{n} x\right)=8^{n} D(x)$ for all $n \in \mathbb{N}$. It follows from (2.3) that

$$
\begin{aligned}
\mu_{C(x)-D(x)}(t) & =\lim _{n \rightarrow \infty} \mu_{\left(C\left(2^{n} x\right) / 8^{n}\right)-\left(D\left(2^{n} x\right) / 8^{n}\right)}(t) \\
\mu_{\left(C\left(2^{n} x\right) / 8^{n}\right)-\left(D\left(2^{n} x\right) / 8^{n}\right)}(t) & \geq \min \left\{\mu_{\left(C\left(2^{n} x\right) / 8^{n}\right)-\left(f\left(2^{n} x\right) / 8^{n}\right)}\left(\frac{t}{2}\right), \mu_{\left(D\left(2^{n} x\right) / 8^{n}\right)-\left(f\left(2^{n} x\right) / 8^{n}\right)}\left(\frac{t}{2}\right)\right\} \\
& \geq \mu_{\varphi\left(2^{n} x, 0\right)}^{\prime}\left(8^{n}(8-\alpha) t\right) \\
& \geq \mu_{\varphi(x, 0)}^{\prime}\left(\frac{8^{n}(8-\alpha) t}{\alpha^{n}}\right) .
\end{aligned}
$$

Since $\lim _{n \rightarrow \infty}\left(8^{n}(8-\alpha) t / \alpha^{n}\right)=\infty$, we get $\lim _{n \rightarrow \infty} \mu_{\varphi(x, 0)}^{\prime}\left(\left(8^{n}(8-\alpha) t\right) / \alpha^{n}\right)=1$. Therefore, it follows that $\mu_{C(x)-D(x)}(t)=1$ for all $t>0$ and so $C(x)=D(x)$. This completes the proof.

Corollary 2.2. Let $X$ be a linear space, $\left(Z, \mu^{\prime}, \min \right)$ an $R N$-space, and $(Y, \mu, \min )$ a complete $R N$ space. Let $p, q$ be nonnegative real numbers and let $z_{0} \in Z$. If $f: X \rightarrow Y$ is a mapping such that

$$
\mu_{f(2 x+y)+f(2 x-y)-2 f(x+y)-2 f(x-y)-12 f(x)}(t) \geq \mu_{\left(\|x\|^{p}+\|y\|^{q}\right) z_{0}}^{\prime}(t), \quad \forall x, y \in X, t>0,
$$

$f(0)=0$ and $p, q<3$, then there exists a unique cubic mapping $C: X \rightarrow Y$ such that

$$
\left.\mu_{f(x)-C(x)}(t) \geq \mu_{\|x\|^{p} z_{0}}^{\prime}\left(2\left(8-2^{p}\right) t\right)\right), \quad \forall x \in X, t>0 .
$$


Proof. Let $\varphi: X \times X \rightarrow Z$ be defined by $\varphi(x, y)=\left(\|x\|^{p}+\|y\|^{q}\right) z_{0}$. Then the proof follows from Theorem 2.1 by $\alpha=2^{p}$.

Corollary 2.3. Let $X$ be a linear space, $\left(Z, \mu^{\prime}, \mathrm{min}\right)$ an $R N$-space, and $(Y, \mu, \mathrm{min})$ a complete $R N$ space. Let $z_{0} \in Z$. If $f: X \rightarrow Y$ is a mapping such that

$$
\mu_{f(2 x+y)+f(2 x-y)-2 f(x+y)-2 f(x-y)-12 f(x)}(t) \geq \mu_{\varepsilon z_{0}}^{\prime}(t), \quad \forall x, y \in X, t>0,
$$

and $f(0)=0$, then there exists a unique cubic mapping $C: X \rightarrow Y$ such that

$$
\mu_{f(x)-C(x)}(t) \geq \mu_{\varepsilon z_{0}}^{\prime}(14 t), \quad \forall x \in X, t>0 .
$$

Proof. Let $\varphi: X \times X \rightarrow Z$ be defined by $\varphi(x, y)=\varepsilon z_{0}$. Then, the proof follows from Theorem 2.1 by $\alpha=1$.

\section{On the stability of quadratic mappings in $\mathbf{R N}$-spaces}

Theorem 3.1. Let $X$ be a linear space, $\left(Z, \mu^{\prime}, \min \right)$ an $R N$-space, and $\varphi: X \times X \rightarrow Z$ a function such that for some $0<\alpha<16$,

$$
\mu_{\varphi(2 x, 0)}^{\prime}(t) \geq \mu_{\alpha \varphi(x, 0)}^{\prime}(t), \quad \forall x \in X, t>0,
$$

$f(0)=0$ and $\lim _{n \rightarrow \infty} \mu_{\varphi\left(2^{n} x, 2^{n} y\right)}^{\prime}\left(16^{n} t\right)=1$ for all $x, y \in X$ and all $t>0$. Let $(Y, \mu, \min )$ be $a$ complete RN-space. If $f: X \rightarrow Y$ is a mapping such that

$$
\mu_{f(2 x+y)+f(2 x-y)-4 f(x+y)-4 f(x-y)-24 f(x)+6 f(y)}(t) \geq \mu_{\varphi(x, y)}^{\prime}(t), \quad \forall x, y \in X, t>0,
$$

then there exists a unique quadratic mapping $Q: X \rightarrow Y$ such that

$$
\left.\mu_{f(x)-Q(x)}(t) \geq \mu_{\varphi(x, 0)}^{\prime}(2(16-\alpha) t)\right)
$$

Proof. From (3.2), it follows that

$$
\begin{aligned}
E_{\lambda, \mu}( & f(2 x+y)+f(2 x-y)-4 f(x+y)-4 f(x-y)-24 f(x)+6 f(y)) \\
& =\inf \left\{t>0: \mu_{f(2 x+y)+f(2 x-y)-4 f(x+y)-4 f(x-y)-24 f(x)+6 f(y)}(t)>1-\lambda\right\} \\
& \leq \inf \left\{t>0: \mu_{\varphi(x, y)}^{\prime}(t)>1-\lambda\right\} \\
& =E_{\lambda, \mu^{\prime}}(\varphi(x, y)), \quad \forall x, y \in X, \lambda \in(0,1) .
\end{aligned}
$$

Putting $y=0$ in (3.4), we get

$$
E_{\lambda, \mu}\left(\frac{f(2 x)}{16}-f(x)\right) \leq \frac{1}{32} E_{\lambda, \mu^{\prime}}(\varphi(x, 0)), \quad \forall x \in X .
$$


Replacing $x$ by $2^{n} x$ in (3.5) and using (3.1), we obtain

$$
\begin{aligned}
E_{\lambda, \mu}\left(\frac{f\left(2^{n+1} x\right)}{16^{n+1}}-\frac{f\left(2^{n} x\right)}{16^{n}}\right) & \leq \frac{1}{32 \times 16^{n}} E_{\lambda, \mu^{\prime}}\left(\varphi\left(2^{n} x, 0\right)\right) \\
& \leq \frac{\alpha^{n}}{32 \times 16^{n}} E_{\lambda, \mu^{\prime}}(\varphi(x, 0)) .
\end{aligned}
$$

It follows from $\left(f\left(2^{n} x\right) / 16^{n}\right)-f(x)=\sum_{k=0}^{n-1}\left(\left(f\left(2^{k+1} x\right) / 16^{k+1}\right)-\left(f\left(2^{k} x\right) / 8^{k}\right)\right)$ and (3.6) that

$$
\begin{aligned}
E_{\lambda, \mu}\left(\frac{f\left(2^{n} x\right)}{16^{n}}-f(x)\right) & =E_{\lambda, \mu}\left(\sum_{k=0}^{n-1}\left(\frac{f\left(2^{k+1} x\right)}{16^{k+1}}-\frac{f\left(2^{k} x\right)}{16^{k}}\right)\right) \\
& \leq \sum_{k=0}^{n-1} E_{\lambda, \mu}\left(\frac{f\left(2^{k+1} x\right)}{16^{k+1}}-\frac{f\left(2^{k} x\right)}{16^{k}}\right) \\
& \leq \sum_{k=0}^{n-1} \frac{1}{32 \times 16^{k}} E_{\lambda, \mu^{\prime}}\left(\varphi\left(2^{k} x, 0\right)\right) \\
& \leq \sum_{k=0}^{n-1} \frac{\alpha^{k}}{32 \times 16^{k}} E_{\lambda, \mu^{\prime}}(\varphi(x, 0)) .
\end{aligned}
$$

Replacing $x$ with $2^{m} x$ in (3.7), we observe that

$$
\begin{aligned}
E_{\lambda, \mu}\left(\frac{f\left(2^{n+m} x\right)}{16^{n+m}}-\frac{f\left(2^{m} x\right)}{16^{m}}\right) & \leq \sum_{k=0}^{n-1} \frac{\alpha^{k}}{32 \times 16^{k+m}} E_{\lambda, \mu^{\prime}}\left(\varphi\left(2^{m} x, 0\right)\right) \\
& \leq \sum_{k=0}^{n-1} \frac{\alpha^{k+m}}{32 \times 16^{k+m}} E_{\lambda, \mu^{\prime}}(\varphi(x, 0)) \\
& \leq \sum_{k=m}^{m+n-1} \frac{\alpha^{k}}{32 \times 16^{k}} E_{\lambda, \mu^{\prime}}(\varphi(x, 0)) \\
& =\frac{E_{\lambda, \mu^{\prime}}(\varphi(x, 0))^{m+n-1}}{32} \sum_{k=m}^{m}\left(\frac{\alpha}{16}\right)^{k} .
\end{aligned}
$$

Then $\left\{f\left(2^{n} x\right) / 16^{n}\right\}$ is a Cauchy sequence in $(Y, \mu, \min )$. Since $(Y, \mu, \min )$ is a complete RNspace, this sequence converges to some point $Q(x) \in Y$. Fix $x \in X$ and put $m=0$ in (3.8). Then we obtain

$$
E_{\lambda, \mu}\left(\frac{f\left(2^{n} x\right)}{16^{n}}-f(x)\right) \leq \frac{E_{\lambda, \mu^{\prime}}(\varphi(x, 0))}{32} \sum_{k=0}^{n-1}\left(\frac{\alpha}{16}\right)^{k}
$$


and so

$$
\begin{aligned}
E_{\lambda, \mu}(Q(x)-f(x)) & \leq E_{\lambda, \mu}\left(Q(x)-\frac{f\left(2^{n} x\right)}{16^{n}}\right)+E_{\lambda, \mu}\left(\frac{f\left(2^{n} x\right)}{16^{n}}-f(x)\right) \\
& \leq E_{\lambda, \mu}\left(Q(x)-\frac{f\left(2^{n} x\right)}{16^{n}}\right)+\frac{E_{\lambda, \mu^{\prime}}(\varphi(x, 0))}{32} \sum_{k=0}^{n-1}\left(\frac{\alpha}{16}\right)^{k} .
\end{aligned}
$$

Taking the limit as $n \rightarrow \infty$ and using (3.10), we get

$$
E_{\lambda, \mu}(Q(x)-f(x)) \leq \frac{E_{\lambda, \mu^{\prime}}(\varphi(x, 0))}{32-2 \alpha},
$$

that is,

$$
\inf \left\{t>0: \mu_{Q(x)-f(x)}(t)>1-\lambda\right\} \leq \inf \left\{t>0: \mu_{\varphi(x, 0)}^{\prime}(2 t(16-\alpha))>1-\lambda\right\}
$$

Then, we have

$$
\mu_{Q(x)-f(x)}(t) \geq \mu_{\varphi(x, 0)}^{\prime}(2 t(16-\alpha))
$$

Replacing $x$ and $y$ by $2^{n} x$ and $2^{n} y$ in (3.2), respectively, we get

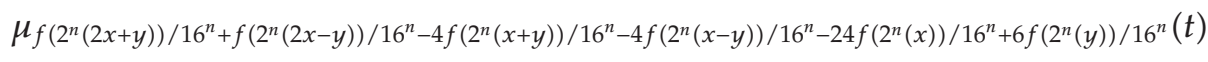

$$
\begin{aligned}
& \geq \mu_{\varphi\left(2^{n} x, 2^{n} y\right)}^{\prime}\left(16^{n} t\right), \quad \forall x, y \in X, t>0 .
\end{aligned}
$$

Since $\lim _{n \rightarrow \infty} \mu_{\varphi\left(2^{n} x, 2^{n} y\right)}^{\prime}\left(16^{n} t\right)=1$, we conclude that $Q$ fulfills (1.2).

To prove the uniqueness of the quadratic mapping $Q$, assume that there exists a quadratic mapping $D: X \rightarrow Y$ which satisfies (3.3). Fix $x \in X$. Clearly, $Q\left(2^{n} x\right)=16^{n} Q(x)$ and $D\left(2^{n} x\right)=16^{n} D(x)$ for all $n \in \mathbb{N}$. It follows from (3.3) that

$$
\begin{aligned}
\mu_{Q(x)-D(x)}(t) & =\lim _{n \rightarrow \infty} \mu_{\left(Q\left(2^{n} x\right) / 16^{n}\right)-\left(D\left(2^{n} x\right) / 16^{n}\right)}(t) \\
\mu_{\left(Q\left(2^{n} x\right) / 16^{n}\right)-\left(D\left(2^{n} x\right) / 16^{n}\right)}(t) & \geq \min \left\{\mu_{\left(Q\left(2^{n} x\right) / 16^{n}\right)-\left(f\left(2^{n} x\right) / 16^{n}\right)}\left(\frac{t}{2}\right), \mu_{\left(D\left(2^{n} x\right) / 8^{n}\right)-\left(f\left(2^{n} x\right) / 8^{n}\right)}\left(\frac{t}{2}\right)\right\} \\
& \geq \mu_{\varphi\left(2^{n} x, 0\right)}^{\prime}\left(16^{n}(16-\alpha) t\right) \\
& \geq \mu_{\varphi(x, 0)}^{\prime}\left(\frac{16^{n}(16-\alpha) t}{\alpha^{n}}\right) .
\end{aligned}
$$

Since $\lim _{n \rightarrow \infty}\left(16^{n}(16-\alpha) t / \alpha^{n}\right)=\infty$, we get $\lim _{n \rightarrow \infty} \mu_{\varphi(x, 0)}^{\prime}\left(16^{n}(16-\alpha) t / \alpha^{n}\right)=1$. Therefore, it follows that $\mu_{Q(x)-D(x)}(t)=1$ for all $t>0$ and so $Q(x) \stackrel{\varphi}{=} D(x)$. This completes the proof. 
Corollary 3.2. Let $X$ be a linear space, $\left(Z, \mu^{\prime}, \mathrm{min}\right)$ an $R N$-space, and $(Y, \mu, \mathrm{min})$ a complete $R N$ space. Let $p, q$ be nonnegative real numbers and let $z_{0} \in Z$. If $f: X \rightarrow Y$ is a mapping such that

$$
\mu_{f(2 x+y)+f(2 x-y)-4 f(x+y)-4 f(x-y)-24 f(x)+6 f(y)}(t) \geq \mu^{\prime}\left(\|x\|^{p}+\|y\|^{q}\right) z_{0}(t), \quad \forall x, y \in X, t>0,
$$

$f(0)=0$ and $p, q<4$, then there exists a unique quadratic mapping $Q: X \rightarrow Y$ such that

$$
\left.\mu_{f(x)-Q(x)}(t) \geq \mu_{\|x\|^{p} z_{0}}^{\prime}\left(2\left(16-2^{p}\right) t\right)\right), \quad \forall x \in X, t>0 .
$$

Proof. Let $\varphi: X \times X \rightarrow Z$ be defined by $\varphi(x, y)=\left(\|x\|^{p}+\|y\|^{q}\right) z_{0}$. Then, the proof follows from Theorem 3.1 by $\alpha=2^{p}$.

Corollary 3.3. Let $X$ be a linear space, $\left(Z, \mu^{\prime}, \mathrm{min}\right)$ an $R N$-space, and $(Y, \mu, \mathrm{min})$ a complete $R N-$ space. Let $z_{0} \in Z$. If $f: X \rightarrow Y$ is a mapping such that

$$
\mu_{f(2 x+y)+f(2 x-y)-4 f(x+y)-4 f(x-y)-24 f(x)+6 f(y)}(t) \geq \mu_{\varepsilon z_{0}}^{\prime}(t), \quad \forall x, y \in X, t>0,
$$

and $f(0)=0$, then there exists a unique quadratic mapping $Q: X \rightarrow Y$ such that

$$
\mu_{f(x)-Q(x)}(t) \geq \mu_{\varepsilon z_{0}}^{\prime}(30 t), \quad \forall x \in X, t>0 .
$$

Proof. Let $\varphi: X \times X \rightarrow Z$ be defined by $\varphi(x, y)=\varepsilon z_{0}$. Then, the proof follows from Theorem 3.1 by $\alpha=1$.

\section{Acknowledgments}

The authors would like to thank the referees for giving useful comments and suggestions for the improvement of this paper. The second author was supported by the Korea Research Foundation Grant funded by the Korean Government (MOEHRD) (KRF-2007-313-C00040).

\section{References}

[1] S. M. Ulam, Problems in Modern Mathematics, John Wiley \& Sons, New York, NY, USA, 1964.

[2] D. H. Hyers, "On the stability of the linear functional equation," Proceedings of the National Academy of Sciences of the United States of America, vol. 27, no. 4, pp. 222-224, 1941.

[3] T. Aoki, "On the stability of the linear transformation in Banach spaces," Journal of the Mathematical Society of Japan, vol. 2, no. 1-2, pp. 64-66, 1950.

[4] Th. M. Rassias, "On the stability of the linear mapping in Banach spaces," Proceedings of the American Mathematical Society, vol. 72, no. 2, pp. 297-300, 1978.

[5] C. Baak and M. S. Moslehian, "On the stability of $J^{*}$-homomorphisms," Nonlinear Analysis: Theory, Methods E Applications, vol. 63, no. 1, pp. 42-48, 2005.

[6] S. Czerwik, Functional Equations and Inequalities in Several Variables, World Scientific, River Edge, NJ, USA, 2002.

[7] D. H. Hyers, G. Isac, and Th. M. Rassias, Stability of Functional Equations in Several Variables, vol. 34 of Progress in Nonlinear Differential Equations and Their Applications, Birkhäuser, Boston, Mass, USA, 1998.

[8] S.-M. Jung, Hyers-Ulam-Rassias Stability of Functional Equations in Mathematical Analysis, Hadronic Press, Palm Harbor, Fla, USA, 2001.

[9] Th. M. Rassias, Ed., Functional Equations, Inequalities and Applications, Kluwer Academic Publishers, Dordrecht, The Netherlands, 2003. 
[10] K.-W. Jun and H.-M. Kim, "The generalized Hyers-Ulam-Rassias stability of a cubic functional equation," Journal of Mathematical Analysis and Applications, vol. 274, no. 2, pp. 867-878, 2002.

[11] K.-W. Jun, H.-M. Kim, and I.-S. Chang, "On the Hyers-Ulam stability of an Euler-Lagrange type cubic functional equation," Journal of Computational Analysis and Applications, vol. 7, no. 1, pp. 21-33, 2005.

[12] J. M. Rassias, "Solution of the Ulam stability problem for quartic mappings," Glasnik Matematički, vol. 34, no. 2, pp. 243-252, 1999.

[13] A. K. Mirmostafaee and M. S. Moslehian, "Fuzzy versions of Hyers-Ulam-Rassias theorem," Fuzzy Sets and Systems, vol. 159, no. 6, pp. 720-729, 2008.

[14] A. K. Mirmostafaee, M. Mirzavaziri, and M. S. Moslehian, "Fuzzy stability of the Jensen functional equation," Fuzzy Sets and Systems, vol. 159, no. 6, pp. 730-738, 2008.

[15] A. K. Mirmostafaee and M. S. Moslehian, "Fuzzy approximately cubic mappings," Information Sciences, vol. 178, no. 19, pp. 3791-3798, 2008.

[16] C. Alsina, "On the stability of a functional equation arising in probabilistic normed spaces," in General Inequalities, 5 (Oberwolfach, 1986), vol. 80 of Internationale Schriftenreihe zur Numerischen Mathematik, pp. 263-271, Birkhäuser, Basel, Switzerland, 1987.

[17] D. Miheț and V. Radu, "On the stability of the additive Cauchy functional equation in random normed spaces," Journal of Mathematical Analysis and Applications, vol. 343, no. 1, pp. 567-572, 2008.

[18] S.-S. Chang, Y. J. Cho, and S. M. Kang, Nonlinear Operator Theory in Probabilistic Metric Spaces, Nova Science, Huntington, NY, USA, 2001.

[19] O. Hadžić and E. Pap, Fixed Point Theory in Probabilistic Metric Spaces, vol. 536 of Mathematics and Its Applications, Kluwer Academic Publishers, Dordrecht, The Netherlands, 2001.

[20] B. Schweizer and A. Sklar, Probabilistic Metric Spaces, North-Holland Series in Probability and Applied Mathematics, North-Holland, New York, NY, USA, 1983.

[21] A. N. Šerstnev, “On the concept of a stochastic normalized space," Doklady Akademii Nauk SSSR, vol. 149 , pp. 280-283, 1963 (Russian). 